\title{
Compact microstrip bandpass filter with sharp passband skirts using square spiral resonators and embedded-resonators
}

\begin{abstract}
The aim of this paper is to produce and develop a new four-pole microstrip bandpass filter (BPF) structure for high selectivity applications. The microstrip BPF is designed using Chebychev lowpass prototype with passband ripple of $0.05 \mathrm{~dB}$ and bandwidth of $120 \mathrm{MHz}$, which operates at center frequency of $2.3 \mathrm{GHz}$. This filter is designed by using square spiral resonator structures and embedded-resonator topology with the same fundamental frequency to make it more compact; furthermore, it has high quality performance in terms of the frequency responses. The size of the compact microstrip filter is $24.74 \times 21.20 \mathrm{~mm} 2$. The proposed filter was designed, fabricated and tested. The measured results show that the minimum passband insertion loss is $2.65 \mathrm{~dB}$, while the measured return loss is better than -11 $\mathrm{dB}$ in the passband. Very good agreement between the simulated and measured results was observed.
\end{abstract}

Keyword: Center frequency; Fundamental frequencies; High quality; High selectivity; Lowpass prototypes; Measured results; Microstrip; Microstrip band-pass filter; Microstrip bandpass filters; Microstrip filter; Pass bands; Passband ripple; Return loss; Spiral resonators 